\title{
TWO WEIGHT INEQUALITIES FOR POSITIVE OPERATORS: DOUBLING CUBES
}

\author{
WEI CHEN AND MICHAEL T. LACEY
}

\begin{abstract}
For the maximal operator $M$ on $\mathbb{R}^{d}$, and $1<p, \rho<\infty$, there is a finite constant $\mathrm{D}=\mathrm{D}_{p, \rho}$ so that this holds. For all weights $w, \sigma$ on $\mathbb{R}^{\mathrm{d}}$, the operator $M(\sigma \cdot)$ is bounded from $\mathrm{L}^{\mathfrak{p}}(\sigma) \rightarrow \mathrm{L}^{\mathfrak{p}}(w)$ if and only the pair of weights $(w, \sigma)$ satisfy the two weight $A_{p}$ condition, and this testing inequality holds:

$$
\int_{\mathrm{Q}} \mathrm{M}\left(\sigma \mathbf{1}_{\mathrm{Q}}\right)^{\mathrm{p}} \mathrm{d} w \lesssim \sigma(\mathrm{Q}),
$$

for all cubes $\mathrm{Q}$ for which there is a cube $\mathrm{P} \supset \mathrm{Q}$ satisfying $\sigma(\mathrm{P})<\operatorname{D\sigma }(\mathrm{Q})$, and $\ell P=\rho \ell Q$. This was recently proved by Kangwei Li and Eric Sawyer. We give a short proof, which is easily seen to hold for several closely related operators.
\end{abstract}

\section{Contents}

1. Introduction 1

2. Proof 2

3. Complements 5

$\begin{array}{ll}\text { References } & 7\end{array}$

\section{INTRODUCTION}

Our subject is the two weight inequalities for the maximal function, fractional integral transforms, and Poisson integrals. For the purposes of this section, we will focus on the maximal function. A weight $w$ is a non-negative Borel measure on $\mathbb{R}^{d}$, and given two weights $w, \sigma$ we say that $(w, \sigma) \in A_{p}$ if the constant

$$
[w, \sigma]_{p}=\sup _{Q}\langle w\rangle_{Q}^{1 / p}\langle\sigma\rangle_{Q}^{1 / p^{\prime}}, \quad p^{\prime}=\frac{p}{p-1} .
$$

The research of W. Chen is supported by the National Natural Science Foundation of China(11771379), the Natural Science Foundation of Jiangsu Province(BK20161326), and the Jiangsu Government Scholarship for Overseas Studies(JS-2017-228). The research of M. T. Lacey is supported in part by grant from the US National Science Foundation, DMS-1600693 and the Australian Research Council ARC DP160100153. 
where here and throughout $\langle w\rangle_{\mathrm{Q}}=|\mathrm{Q}|^{-1} \int_{\mathrm{Q}} w \mathrm{~d} x$. With this notation the maximal function is

$$
M f=\sup _{\text {Qcube }}\langle f\rangle_{\mathrm{Q}} \mathbf{1}_{\mathrm{Q}}
$$

The classical two weight inequality for the maximal function due to Sawyer [14] is below. It shows that the inequality for the maximal function reduces to a testing inequality, for indicators of cubes.

Theorem 1.2. For two weights $(\mathcal{w}, \sigma)$ we have the inequality

$$
\|M(\sigma f)\|_{L^{p}(w)} \lesssim\|f\|_{L^{p}(\sigma)}
$$

if and only if the testing inequality below holds:

$$
\sup _{\mathrm{Q}: \sigma(\mathrm{Q})>0} \sigma(\mathrm{Q})^{-1 / \mathrm{p}}\left\|\mathbf{1}_{\mathrm{Q}} \mathrm{M}\left(\sigma \mathbf{1}_{\mathrm{Q}}\right)\right\|_{\mathrm{Lp}^{\mathrm{p}}(w)}<\infty .
$$

Recent papers Li and Sawyer $[10,11]$ began a study of a weaker class of testing inequalities in the two weight setting. (Their papers include interesting motivation and background.) They introduce four such conditions. The definition below is weaker than their weakest condition: Test the maximal function on indicators of cubes $\mathrm{Q}$ which have some parent on which $\sigma$ is doubling.

Definition 1.3. Given two weights $(\mathcal{w}, \sigma)$, and $1<p, \rho, \mathrm{D}<\infty$ we say that $(\mathcal{w}, \sigma)$ satisfy a $(p, \rho, D)$ parent doubling testing condition if there is a positive finite constant $\mathfrak{P}=\mathfrak{P}_{\rho, \mathrm{D}}=\mathfrak{P}(\mathcal{w}, \sigma, d, p, \rho, D)$ so that we have

$$
\left\|\mathbf{1}_{\mathrm{Q}} M\left(\sigma \mathbf{1}_{\mathrm{Q}}\right)\right\|_{\mathrm{Lp}(w)} \leq \mathfrak{P} \sigma(\mathrm{Q})^{1 / p},
$$

for every cube $Q$ for which there is a second cube $P \supset Q$, with $\ell P \geq \rho \ell Q$, and $\sigma(P) \leq$ $\mathrm{D} \sigma(\mathrm{Q})$.

Above $\ell Q=|Q|^{1 / d}$ is the side length of $Q$. The case of $\rho=p=2$ below is (just a little stronger than) the main result of $\mathrm{Li}$ and Sawyer [11].

Theorem 1.5. Let $1<p, \rho<\infty$. There is a constant $\mathrm{D}=\mathrm{D}_{\mathrm{d}, \mathrm{p}, \rho}$ so that for any pair of weights $(\sigma, w)$ we have

$$
\|M(\sigma \cdot)\|_{L^{p}(\sigma) \rightarrow L^{p}(w)} \simeq[w, \sigma]_{p}+\mathfrak{P}_{\rho, \mathrm{D}} .
$$

Our proof is short and readily adapts to several closely related operators, as indicated in the concluding section.

\section{PROOF}

The norm bound on $M$ easily implies the two weight $A_{p}$ condition on the weights, as well as the testing condition for all cubes, not just those with a doubling parent. The content of the Theorem is that the reverse implication holds. 
Our theorem only claims that there is a sufficiently large doubling parameter D which can be used for all pairs of weights $(w, \sigma)$. Below, we will consider values of $1<\rho \leq 2$. For integers $n=3,4, \ldots$, , and choices of $n-1<\rho \leq n$, the argument proceeds by replacing the dyadic grids introduced below by $n$-ary grids. We omit the details.

By a dyadic grid we need a collection $\mathcal{D}$ of cubes in $\mathbb{R}^{\mathrm{d}}$ for which (a) if $\mathrm{P}, \mathrm{Q} \in \mathcal{D}$, then $P \cap Q$ is empty, $P$ or $Q$, and (b) for all integers $k$, the cubes $\left\{Q \in \mathcal{D}:|Q|=2^{d k}\right\}$ partition $\mathbb{R}^{\mathrm{d}}$. Associated to the grid $\mathcal{D}$ is the maximal function

$$
M_{\mathcal{D}} f=\sup _{\mathrm{Q} \in \mathcal{D}}\langle f\rangle_{\mathrm{Q}} \mathbf{1}_{\mathrm{Q}}
$$

As is well known, there are a finite number of grids $\mathcal{D}_{1}, \ldots, \mathcal{D}_{3^{\mathrm{d}}}$ for which

$$
M f \lesssim \sup _{1 \leq j \leq 3^{\mathrm{d}}} M_{\mathcal{D}_{\mathrm{j}}} f
$$

Set $D=2^{d \frac{p+1}{p-1}}$. It suffices to show that under the two weight $A_{p}$ and $(p, 2, D)$ parent testing condition, for any dyadic grid $\mathcal{D}$, the maximal function $M_{\mathcal{D}}(\sigma \cdot)$ is bounded from $\mathrm{L}^{\mathrm{p}}(\sigma)$ to $\mathrm{L}^{\mathrm{p}}(w)$.

Sawyer's Theorem 1.2 holds for $M_{\mathcal{D}}$. Namely, it suffices to show that for any cube $\mathrm{Q}_{0} \in \mathcal{D}$, we have

$$
\int_{\mathrm{Q}_{0}} \mathrm{M}_{\mathcal{D}}\left(\sigma \mathbf{1}_{\mathrm{Q}_{0}}\right)^{\mathrm{p}} \mathrm{d} w \lesssim\left([w, \sigma]_{\mathrm{p}}^{\mathrm{p}}+\mathfrak{P}^{\mathrm{p}}\right) \sigma\left(\mathrm{Q}_{0}\right) .
$$

We are free to restrict the supremum to the collection of cubes $\mathcal{Q}=\left\{\mathrm{Q} \in \mathcal{D}: \mathrm{Q} \subset \mathrm{Q}_{0}\right\}$ of cubes contained in $Q_{0}$.

Partition $\mathcal{Q}$ into four subcollections using these definitions.

- (Testing Collection) Let $\mathcal{T}^{*}$ be the maximal elements $\mathrm{Q} \in \mathcal{D}$ with $\mathrm{Q} \subset \mathrm{Q}_{0}$ so that the testing inequality (1.4) holds. Set $\mathcal{T}_{\mathrm{Q}}=\{\mathrm{P} \in \mathcal{Q}: \mathrm{P} \subset \mathrm{Q}\}$, for $\mathrm{Q} \in \mathcal{T}^{*}$. And set $\mathcal{T}=\bigcup_{\mathrm{Q} \in \mathcal{T} *} \mathcal{T}_{\mathrm{Q}}$.

- (The Top) Let $\mathcal{U}=\left\{\mathrm{Q} \in \mathcal{Q} \backslash \mathcal{T}: 2^{\mathrm{k}} \ell \mathrm{Q} \geq \ell \mathrm{Q}_{0}\right\}$. We choose $\mathrm{k}$ large enough that $2^{\mathrm{dk}} k^{-p}>1$. These are the cubes which are close to the top cube $\mathrm{Q}_{0}$.

- (Small $A_{p}$ Cubes) Let $\mathcal{A}$ be those cubes $\mathrm{Q} \in \mathcal{Q} \backslash(\mathcal{T} \cup \mathcal{U})$ such that

$$
\langle\sigma\rangle_{\mathrm{Q}}^{1 / \mathrm{p}^{\prime}}\langle w\rangle_{\mathrm{Q}}^{1 / \mathrm{p}} \leq \frac{[w, \sigma]_{\mathrm{p}}}{\log \ell \mathrm{Q}_{0} / \ell \mathrm{Q}}
$$

That is, the local $A_{p}$ constant at $Q$ is very small.

- (Remaining Cubes) Let $\mathcal{R}=\mathcal{Q} \backslash(\mathcal{T} \cup \mathcal{U} \cup \mathcal{A})$. 
We show that the maximal function over each collection satisfies the testing inequality (2.2). The Testing Collection is very easy:

$$
\begin{aligned}
\int_{\mathrm{Q}_{0}} \sup _{\mathrm{Q} \in \mathcal{T}}\langle\sigma\rangle_{\mathrm{Q}}^{\mathrm{p}} \mathbf{1}_{\mathrm{Q}} \mathrm{d} w & \leq \sum_{\mathrm{Q} \in \mathcal{T}^{*}} \int_{\mathrm{Q}} \mathrm{M}\left(\sigma \mathbf{1}_{\mathrm{Q}}\right)^{\mathrm{p}} \mathrm{d} w \\
& \leq \mathfrak{P}_{2, \mathrm{D}}^{\mathrm{p}} \sum_{\mathrm{Q} \in \mathcal{T}^{*}} \sigma(\mathrm{Q}) \leq \mathfrak{P}^{\mathrm{p}} \sigma\left(\mathrm{Q}_{0}\right) .
\end{aligned}
$$

The Top Collection $\mathcal{U}$ has at most $2^{1+\mathrm{d}(\mathrm{k}+1)}$ elements, and we just use the $A_{p}$ condition to see that

$$
\begin{aligned}
\int_{\mathrm{Q}_{0}} \sup _{\mathrm{Q} \in \mathcal{U}}\langle\sigma\rangle_{\mathrm{Q}}^{\mathrm{p}} \mathbf{1}_{\mathrm{Q}} \mathrm{d} w & \leq \sum_{\mathrm{Q} \in \mathcal{U}}\langle\sigma\rangle_{\mathrm{Q}}^{\mathrm{p}}\langle w\rangle_{\mathrm{Q}} \\
& \leq[w, \sigma]_{\mathfrak{p}}^{\mathrm{p}} \sum_{\mathrm{Q} \in \mathcal{U}} \sigma(\mathrm{Q}) \lesssim[w, \sigma]_{\mathrm{p}}^{\mathrm{p}} \sigma\left(\mathrm{Q}_{0}\right) .
\end{aligned}
$$

The implied constant depends upon $k$, but that is a fixed integer.

The Small $A_{p}$ Cubes are also trivially sum up, using the condition in (2.3).

$$
\begin{aligned}
\int_{\mathrm{Q}_{0}} \sup _{\mathrm{Q} \in \mathcal{A}}\langle\sigma\rangle_{\mathrm{Q}}^{\mathrm{p}} \mathbf{1}_{\mathrm{Q}} \mathrm{d} w & \leq \sum_{\mathrm{Q} \in \mathcal{A}}\langle\sigma\rangle_{\mathrm{Q}}^{\mathrm{p}}\langle w\rangle_{\mathrm{Q}} \\
& \leq[w, \sigma]_{\mathrm{p}}^{\mathrm{p}} \sum_{\mathrm{Q} \in \mathcal{A}} \frac{\sigma(\mathrm{Q})}{\left[\log \ell \mathrm{Q}_{0} / \ell \mathrm{Q}\right]^{\mathrm{p}}} \lesssim[w, \sigma]_{\mathrm{p}}^{\mathrm{p}} \sigma\left(\mathrm{Q}_{0}\right) .
\end{aligned}
$$

Thus, the core of the argument is control of the Remaining Cubes, $\mathcal{R}$. Indeed, we claim that this collection is empty, since a cube that has a large local $A_{p}$ product is also approximately doubling.

Suppose $\mathcal{R} \neq \emptyset$. Thus, there is a cube $\mathrm{Q} \subset \mathrm{Q}_{0}$, which satisfies $\ell \mathrm{Q}<2^{-\mathrm{k}} \ell \mathrm{Q}_{0}$, fails (2.3), and no ancestor of $\mathrm{Q}$ also contained inside of $\mathrm{Q}_{0}$, has a doubling parent. The last condition is very strong.

Let $\mathrm{Q}^{(1)}$ be the $\mathcal{D}$-parent of $\mathrm{Q}$, and let $\mathrm{Q}^{(\mathrm{k}+1)}=\left(\mathrm{Q}^{(\mathrm{k})}\right)^{(1)}$. Define integer $\mathrm{m}$ by $\mathrm{Q}_{0}=\mathrm{Q}^{(\mathrm{m})}$. For any integer $0 \leq \mathrm{k}<\mathrm{m}$, we necessarily have $\sigma\left(\mathrm{Q}^{(\mathrm{k}+1)}\right) \geq \operatorname{D} \sigma\left(\mathrm{Q}^{(\mathrm{k})}\right)$, since $\mathrm{Q}^{(\mathrm{k}+1)}$ is a $\rho$-parent of $\mathrm{Q}^{(\mathrm{k})}$. That is, $\sigma\left(\mathrm{Q}_{0}\right) \geq \mathrm{D}^{\mathrm{m}} \sigma(\mathrm{Q})$. From this, we see that $m$ cannot be very large.

$$
\begin{aligned}
{[w, \sigma]_{\mathrm{p}}^{\mathrm{p}} } & \geq\langle\sigma\rangle_{\mathrm{Q}_{0}}^{\mathrm{p}-1}\langle w\rangle_{\mathrm{Q}_{0}} \\
& \geq \mathrm{D}^{\mathrm{m}(\mathrm{p}-1)}\left[\frac{\sigma(\mathrm{Q})}{\left|\mathrm{Q}^{(\mathrm{m})}\right|}\right]^{\mathrm{p}-1} \frac{w(\mathrm{Q})}{\left|\mathrm{Q}^{(\mathrm{m})}\right|} \\
& \geq\left[\mathrm{D} / 2^{\mathrm{dp^{ \prime }}}\right]^{\mathrm{m}(\mathrm{p}-1)}\langle\sigma\rangle_{\mathrm{Q}}^{\mathrm{p}-1}\langle w\rangle_{\mathrm{Q}} \\
& \geq[w, \sigma]_{\mathrm{p}}^{\mathrm{p}}\left[\mathrm{D} / 2^{\mathrm{dp^{ \prime }}}\right]^{\mathrm{m}(\mathrm{p}-1)} \mathrm{m}^{-\mathrm{p}} \geq[w, \sigma]_{\mathrm{p}}^{\mathrm{p}} 2^{\mathrm{dm}} \mathrm{m}^{-\mathrm{p}}
\end{aligned}
$$


The constants are explicit, and the last line follows by choice of $\mathrm{D}$. We see that $m<k$. That is, the cube is in the collection $\mathcal{U}$, which is a contradiction.

\section{Complements}

1. The conditions in Theorem 1.5 can be strengthened by adding the condition that the doubling cubes satisfy $\sigma(\partial D)=0$. This is accomplished by selection of random grids. The discussion needed is given by Li and Sawyer [11, §2], and we omit the details. Similar comments apply to the extensions we mention below.

2. Theorem 1.5 has a straight forward extension to fractional maximal functions.

3. The method of proof easily extends to other operators which are well approximated by dyadic grids. One of these is the Poisson integral given by

$$
\operatorname{Pf}(x, t)=\int \frac{t}{\left(t^{2}+|x-y|^{2}\right)^{\frac{d+1}{2}}} f(y) d y, \quad t>0
$$

Given weights $\sigma$ on $\mathbb{R}^{\mathrm{d}}$ and $\mathcal{w}$ on the upper half space $\mathbb{R}_{+}^{\mathrm{d}+1}$, we remark that the role of cubes in $\mathbb{R}_{+}^{\mathrm{d}+1}$ are played by Carleson cubes, namely $\tilde{Q}=\mathrm{Q} \times[0, \ell \mathrm{Q})$, for $\mathrm{Q} \subset \mathbb{R}^{\mathrm{d}}$. The definition of the two weight $A_{p}$ condition is then

$$
[w, \sigma]_{\mathrm{p}}=\sup _{\mathrm{Q}}\langle w\rangle_{\tilde{\mathrm{Q}}}^{1 / \mathrm{p}}\langle\sigma\rangle_{\mathrm{Q}}^{1 / \mathrm{p}^{\prime}}
$$

Using similar methods, one can prove this version of the Poisson two weight theorem of Sawyer [13]. We single out this statement since the Sawyer's Poisson theorem is an important ingredient of the two weight inequality for the Hilbert transform $[6,8]$.

Theorem 3.2. Let $1<p, \rho<\infty$. There is a $\mathrm{D}>1$ so that this holds. Let $w$ be a weight on $\mathbb{R}_{+}^{\mathrm{d}}$, $\sigma$ on $\mathbb{R}^{\mathrm{d}}$. These conditions are necessary and sufficient for $\mathrm{P}(\sigma \cdot)$ to map $\mathrm{L}^{\mathrm{p}}\left(\mathbb{R}^{\mathrm{d}}, \sigma\right)$ to $\mathrm{L}^{\mathrm{p}}\left(\mathbb{R}_{+}^{\mathrm{d}+1}, w\right)$. There is a finite constant $\mathfrak{P}$ so that

(1) The $A_{p}$ condition (3.1) holds.

(2) If $\mathrm{Q} \subset \mathbb{R}^{\mathrm{d}}$ is a cube for which $\sigma(\rho \mathrm{Q})<\mathrm{D} \sigma(\mathrm{Q})$, then, $\left\|\mathbf{1}_{\tilde{Q}} \mathrm{P}\left(\sigma \mathbf{1}_{\mathrm{Q}}\right)\right\|_{\mathrm{L}^{\mathrm{p}}\left(\mathbb{R}_{+}^{\mathrm{d}+1}, w\right)} \leq$ $\mathfrak{P} \sigma(\mathrm{Q})^{1 / p}$.

(3) If $\mathrm{Q} \subset \mathbb{R}^{\mathrm{d}}$ is a cube for which $w(\widetilde{\rho Q})<\mathrm{D} \sigma(\tilde{\mathrm{Q}})$, then, $\left\|\mathbf{1}_{\mathrm{Q}} \mathrm{P}^{*}\left(w \mathbf{1}_{\tilde{\mathrm{Q}}}\right)\right\|_{\mathrm{L}^{p^{\prime}\left(\mathbb{R}^{\mathrm{d}}, \sigma\right)}} \leq$ $\mathfrak{P} \mathcal{W}(\tilde{\mathrm{Q}})^{1 / p^{\prime}}$.

Let us briefly indicate the proof. For any dyadic grid $\mathcal{D}$, we can define

$$
\mathrm{P}_{\mathcal{D}} \mathrm{f}=\sum_{\mathrm{Q} \in \mathcal{D}}\langle\mathrm{f}\rangle_{\mathrm{Q}} \mathbf{1}_{\mathrm{Q}}
$$

One has $P_{\mathcal{D}} f \lesssim P f$, but also an analog of (2.1) holds. That is, there are finitely many dyadic grids $\mathcal{D}_{1}, \ldots, \mathcal{D}_{3 \text { d }}$ for which

$$
\text { Pf } \lesssim \sup _{1 \leq j \leq 3^{\mathrm{d}}} P_{\mathcal{D}_{j}} f .
$$


It therefore remains to see that the three conditions in Theorem 3.2 imply that $P_{\mathcal{D}}$ is bounded, for any choice of grid.

There is a Sawyer type testing theorem for dyadic positive operators [9], so that it suffices to verify the testing inequality

$$
\left\|\mathbf{1}_{\tilde{Q}} \mathrm{P}_{\mathcal{D}}\left(\sigma \mathbf{1}_{\mathrm{Q}}\right)\right\|_{L^{p}(w)} \lesssim\left([w, \sigma]_{\mathrm{p}}+\mathfrak{P}\right) \sigma(\mathrm{Q})^{1 / p} .
$$

as well as the dual estimate. We have arrived at the point (2.2) in our proof of Theorem 1.5. The remaining steps easily extend to this setting.

4. One can also deduce a doubling parent testing type condition for the fractional integral operators

$$
T_{\alpha} f=\int f(x-y) \frac{d y}{|y|^{d \alpha}}, \quad 0<\alpha<1 .
$$

Theorem 3.3. Let $1<\rho<\infty$ and $1<p \leq q<\infty$. There is a D $>1$ so that this holds. Let $(\mathcal{w}, \sigma)$ be weights on $\mathbb{R}^{\mathrm{d}}$. These conditions are necessary and sufficient for $\mathrm{P}(\sigma \cdot)$ to map $\mathrm{L}^{\mathrm{p}}\left(\mathbb{R}^{\mathrm{d}}, \sigma\right)$ to $\mathrm{L}^{\mathrm{q}}\left(\mathbb{R}^{\mathrm{d}}, w\right)$. There is a finite constant $\mathfrak{P}$ so that

(1) The pair of weights satisfy the $A_{p, q}$ condition $\sup _{Q} \frac{w(Q)^{1 / q} \sigma(Q)^{1 / p^{\prime}}}{|Q|^{\alpha}}$.

(2) If $\mathrm{Q} \subset \mathbb{R}^{\mathrm{d}}$ is a cube for which $\sigma(\rho \mathrm{Q})<\mathrm{D} \sigma(\mathrm{Q})$, then, $\left\|\mathbf{1}_{\mathrm{Q}} \mathrm{T}\left(\sigma \mathbf{1}_{\mathrm{Q}}\right)\right\|_{\mathrm{Lq}(w)} \leq$ $\mathfrak{P} \sigma(\mathrm{Q})^{1 / p}$.

(3) If $\mathrm{Q} \subset \mathbb{R}^{\mathrm{d}}$ is a cube for which $w(\rho \mathrm{Q})<\mathrm{Dw}(\mathrm{Q})$, then, $\left\|\mathbf{1}_{\mathrm{Q}} \mathrm{T}\left(w \mathbf{1}_{\mathrm{Q}}\right)\right\|_{\mathrm{L}^{\mathrm{p}^{\prime}(\sigma)}} \leq$ $\mathfrak{P} w(\mathrm{Q})^{1 / \mathrm{q}^{\prime}}$.

There is a corresponding characterization of the weak type inequality. The sketch of Theorem 3.2 applies to the Theorem above.

5. It is an interesting question to gain information about the optimal choice of $D=D_{p, \rho}$ in Theorem 1.5. We have not sought to do so, and comment briefly on the case of $\rho=p=2$. It is clear that $D$ cannot be very small, because then the allowed cubes on which one tests the norm of the maximal function are just too few, or subcritical for the pair of weights.

For instance, one knows that for weights $w \in A_{2}$ that one has

$$
\|M\|_{L^{2}(w) \rightarrow L^{2}(w)} \lesssim[w]_{A_{2}} .
$$

This estimate is sharp in the power of the $A_{2}$ constant, as is shown by considering the weight $w(x)=|x|^{\mathrm{d}-\epsilon}$ for $0<\epsilon<1 / 2$. One can calculate that $[w]_{\mathrm{A}_{2}} \simeq \epsilon^{-1}$, with the cubes that demonstrate this being those centered at the origin. Note that with cube $Q$ centered at the origin, one has

$$
w(\mathrm{Q}) \simeq(\ell \mathrm{Q})^{2 \mathrm{~d}-\epsilon}
$$

It follows that the best possible choice of $\mathrm{D}=\mathrm{D}_{2,2}$ would have to be of the order of $2^{2 \mathrm{~d}}$. We have shown that $D=2^{3 \mathrm{~d}}$ is sufficient. 
6. For the values of $1<\rho<2$, we are providing a very poor estimate of $D_{\rho, p}$. Indeed, one would suspect that $D_{\rho, p} \rightarrow 1$ as $\rho \downarrow 1$. To show this, one would seem to need an improved notion of a shifted grids. The appendix of [1] gives one suggestion. Similar sorts of questions have been addressed in [2,3].

7. It is of interest to extend the results of this paper to non-positive operators. One easy remark is this. Let $\mathcal{D}$ be a dyadic grid in $\mathbb{R}^{\mathrm{d}}$, and let $\left\{\Delta_{\mathrm{Q}}: \mathrm{Q} \in \mathcal{D}\right\}$ be the associated martingale differences. Define a martingale transform by

$$
\mathrm{Tf}=\sum_{\mathrm{Q} \in \mathcal{D}} \epsilon_{\mathrm{Q}} \Delta_{\mathrm{Q}} \mathrm{f}, \quad \epsilon_{\mathrm{Q}} \in\{ \pm 1\} .
$$

For the two weight inequality, one has the result of Nazarov-Treil-Volberg [12] in the $\mathrm{L}^{2}$ case. This leads to the following sufficient conditions for a two weight inequality.

Theorem 3.4. Let $(w, \sigma)$ be weights on $\mathbb{R}^{\mathrm{d}}$ which satisfy the $A_{2}$ condition (1.1). There is a constant $\mathrm{D}>1$ so that these two conditions are sufficient conditions for $\mathrm{T}(\sigma \cdot)$ to map $\mathrm{L}^{2}(\sigma)$ to $\mathrm{L}^{2}(w)$ : For some finite constant $\mathfrak{P}$,

(1) For all cubes $\mathrm{Q} \in \mathcal{D}$ with $\sigma\left(\mathrm{Q}^{(1)}\right)<\operatorname{D\sigma }(\mathrm{Q})$, there holds $\left\|\mathbf{1}_{\mathrm{Q}} \mathrm{T}\left(\sigma \mathbf{1}_{\mathrm{Q}}\right)\right\|_{\mathrm{L}^{2}(w)} \leq$ $\mathfrak{P} \sigma(\mathrm{Q})^{1 / 2}$.

(2) The same condition above holds, with the roles of $\sigma$ and $w$ reversed.

Above, $\mathrm{Q}^{(1)}$ is the dyadic parent of $\mathrm{Q}$.

We state this in the case of $p=2$, as the $L^{p}$-case is much more complicated, see Vuorinen [15]. The weak-type inequality for maximal truncations of martingale transforms does admit an testing characterization. See [4, Thm 4.3]. One can consult [5] for information about the continuous case.

8. Certain kinds of g-functions have a two weight characterization [7]. That theorem can probably be relaxed to the current setting. More involved would be the weak-type estimate for maximal truncations of singular integrals, characterized in [4].

9. Potentially more interesting is relaxing the testing conditions in the two weight inequality for the Hilbert transform $[6,8]$. It seems very likely that such a result is true.

\section{REFERENCES}

[1] Sarah H. Ferguson and Michael T. Lacey, A characterization of product BMO by commutators, Acta Math. 189 (2002), no. 2, 143-160. MR1961195

[2] Paul Hagelstein, Teresa Luque, and loannis Parissis, Tauberian conditions, Muckenhoupt weights, and differentiation properties of weighted bases, Trans. Amer. Math. Soc. 367 (2015), no. 11, 79998032. MR3391907

[3] Paul Hagelstein and loannis Parissis, Weighted Solyanik estimates for the Hardy-Littlewood maximal operator and embedding of $\mathcal{A}_{\infty}$ into $\mathcal{A}_{\mathrm{p}}$, J. Geom. Anal. 26 (2016), no. 2, 924-946. MR3472823

[4] Tuomas P. Hytönen, Michael T. Lacey, Henri Martikainen, Tuomas Orponen, Maria Carmen Reguera, Eric T. Sawyer, and Ignacio Uriarte-Tuero, Weak and strong type estimates for maximal truncations of Calderón-Zygmund operators on $A_{p}$ weighted spaces, J. Anal. Math. 118 (2012), no. 1, 177-220. MR2993026 
[5] Michael Lacey, Eric T. Sawyer, and Ignacio Uriarte-Tuero, A characterization of two weight norm inequalities for maximal singular integrals with one doubling measure, Anal. PDE 5 (2012), no. 1, 1-60. MR2957550

[6] Michael T. Lacey, Two-weight inequality for the Hilbert transform: a real variable characterization, II, Duke Math. J. 163 (2014), no. 15, 2821-2840. MR3285858

[7] Michael T. Lacey and Kangwei Li, Two weight norm inequalities for the g function, Math. Res. Lett. 21 (2014), no. 3, 521-536. MR3272028

[8] Michael T. Lacey, Eric T. Sawyer, Chun-Yen Shen, and Ignacio Uriarte-Tuero, Two-weight inequality for the Hilbert transform: a real variable characterization, I, Duke Math. J. 163 (2014), no. 15, 27952820. MR3285857

[9] Michael T. Lacey, Eric T. Sawyer, and Ignacio Uriarte-Tuero, Two Weight Inequalities for Discrete Positive Operators, ArXiv e-prints (November 2009), arXiv:0911.3437, available at 0911.3437.

[10] Kangwei Li and Eric T. Sawyer, Restricted testing for positive operators, ArXiv e-prints (September 2018), arXiv:1809.04873, available at 1809.04873.

[11] _ Restricted testing for the Hardy-Littlewood maximal function, ArXiv e-prints (November 2018), arXiv:1811.11032, available at 1811.11032.

[12] Fedor Nazarov, Serguei Treil, and Alexander Volberg, Two weight inequalities for individual Haar multipliers and other well localized operators, Math. Res. Lett. 15 (2008), no. 3, 583-597. MR2407233

[13] Eric T. Sawyer, A two weight weak type inequality for fractional integrals, Trans. Amer. Math. Soc. 281 (1984), no. 1, 339-345. MR719674

[14] _ A characterization of a two-weight norm inequality for maximal operators, Studia Math. 75 (1982), no. 1, 1-11. MR676801

[15] Emil Vuorinen, $\mathrm{L}^{\mathrm{p}}(\mu) \rightarrow \mathrm{L}^{\mathrm{q}}(\boldsymbol{v})$ characterization for well localized operators, J. Fourier Anal. Appl. 22 (2016), no. 5, 1059-1075. MR3547711

School of Mathematical Sciences, Yangzhou University, Yangzhou 225002, China; School of Mathematics, Georgia Institute of Technology, Atlanta GA 30332, USA

E-mail address: weichen@yzu.edu.cn

School of Mathematics, Georgia Institute of Technology, Atlanta GA 30332, USA

E-mail address: lacey@math.gatech.edu 